\title{
Comparison of objective and subjective assessments for perception of facial symmetry
}

\author{
Ayla Kürkçüoğlu' ${ }^{1}$ Ozan L. Abbas², Deniz Mert Ayan ${ }^{3}$, Rana Baykan $^{3}$, Ecem Demirkan ${ }^{3}$, Ödül Özkan ${ }^{3}$, \\ Irmak Özkubat ${ }^{3}$, Mert Şimşek ${ }^{3}$ \\ ${ }^{1}$ Department of Anatomy, School of Medicine, Baskent University, Ankara, Turkey \\ ${ }^{2}$ Department of Plastic and Reconstructive Surgery, School of Medicine, Abi Evran University, Kurşehir, Turkey \\ ${ }^{3} 3 r d$ Year Student, School of Medicine, Baskent University, Ankara, Turkey
}

\begin{abstract}
Objectives: Symmetrical patterns of the face are generally preferred by the viewers. However, the process of perception of symmetry is far from perfect and is affected by subjective factors. In this study, we aimed to demonstrate the differences between objective and subjective assessments for perception of facial asymmetry.

Methods: For objective evaluation, the anteroposterior photographs of 450 volunteers were analyzed anthropometrically for symmetry. Subjective evaluations were conducted by seven individuals who assessed each face based solely on symmetry.

Results: We found a difference between objective measurements and subjective evaluations. No statistically significant difference concerning the rates derived from anthropometric points was found between sexes. After the comparison of these rates, no subject was found to have a perfectly symmetrical face, and all subjects showed some asymmetry in one or more of their anthropometric points.

Conclusion: With subjective evaluation, asymmetry was found most commonly in the middle third of the face. Nose and alar margins may be the determining points for the observer during subjective evaluation, thus we think the best region to infer asymmetry from is the middle third of the face. Because of this, thousands of patients complain of asymmetrical noses and undergo rhinoplastic surgery every year. This study also demonstrates individual differences in subjective evaluations. These differences and lack of objectively symmetrical faces for every set of points are very important for patients requesting plastic surgery procedures such as rhinoplasty out of aesthetic concerns.
\end{abstract}

Keywords: facial asymmetry; objective assessment; subjective assessment

Anatomy 2016;10(2):94-98 @2016 Turkish Society of Anatomy and Clinical Anatomy (TSACA)

\section{Introduction}

Facial beauty, considered as the most prominent determinant of perception of beauty, is closely related to the sex, gender, age and race of the individual. It was believed that the perception of attractiveness and beauty was formed according to the social norms of the culture of the individual, as in the saying "beauty is in the eye of the beholder". ${ }^{[1]}$ However, later studies have found that certain criteria such as symmetry, averageness, sexual dimorphism and adherence to ideal ratios are common between cultures. ${ }^{[2]}$

Evaluation of the facial beauty by the human brain is a very fast cognitive process; however, this process is far from perfect and is affected by subjective factors. Several studies have been conducted in order to associate the subjective perception of attractiveness with objective facial values such as height, ratio, angle and symmetry. ${ }^{[3-5]}$ In this context, facial asymmetry has been perceived as a major determinant of attractiveness.

Anthropometric measurements help surgeons evaluate deformities objectively and make operative assessments. ${ }^{[6]}$ However, the difference between subjective and objective assessments may cause dissatisfaction with facial plastic surgery. In this study, we aimed to demonstrate the difference between the objective and subjective assessments for perception of facial asymmetry. 


\section{Materials and Methods}

This study was approved by the Başkent University Ethics Committee on Human Research (KA14/32618, registered 2014-12). 450 subjects (229 males, 221 females) aged between 18 and 25 years (for females, 23 years \pm 4.0 ; for males 24 years \pm 3.8 ) volunteered for this study. Exclusion criteria were presence of congenital craniofacial anomalies, history of trauma or previous orthodontic, orthognathic, or prosthodontic treatment. All faces were photographed from the front, in a seated position, $110 \mathrm{~cm}$ away from the camera. Tripod was adjusted for each subject's height, keeping the camera horizontal. Subjects' hair was put behind their ears in order to reveal the forehead and hairline, and they were asked to remove their glasses. Subjects with facial hair or make-up which would prevent accurate detection of facial lines or features were excluded from this study. Each subject was asked to relax, close their lips slightly and naturally, and express no emotions. Photographs were taken using a 16.4 megapixel Nikon D7000 camera body and $18-105 \mathrm{~mm}$ f/5.3 NIKKOR lens, with values of ISO $2000, \mathrm{f} / 5.3,70 \mathrm{~mm}( \pm 10 \mathrm{~mm})$. Adobe Nikon AF-S DX NIKKOR 18-105 f/3.5-5.6G ED VR lens correction profile was applied to each photograph to minimize lens distortion.

\section{Objective measurements}

All photographs were stored, marked and measured digitally. Photographs were marked using the Adobe Photoshop CS6 software (Adobe Inc.; 2012; San Jose, $\mathrm{CA})$. A 5 px round brush tool was used for anthropometric points and the line tool was used for midline. Each photograph was uploaded to the Analyzing Digital Images software (2014) developed by John Pickle (Concord Academy; formerly, Museum of Science, Boston), and updated by Dan Gullage (STEM Education Institute, University of Massachusetts Amherst). This software has the option for marking a scale to use as a metric reference, if one is present in the image. If no scale is present, measurements can be performed using pixels. In our case, a scale was present, marked and used as a reference point. However, as this study used rates instead of lengths, units were omitted. Ten anthropometric points used to measure symmetry in this study were the lateral canthi (LC), medial canthi (MC), zygoma points (Z), alar margins (AM) and oral commissures (OC) (Figure 1). All measurements were performed by the same researcher.

A midline (ML) was drawn on each photograph, traversing at least two of the following three points: the central hairline point, central point of the nasal bridge and bottom of the chin. The lengths from the ten determined points on one side of the face to the midline were meas-

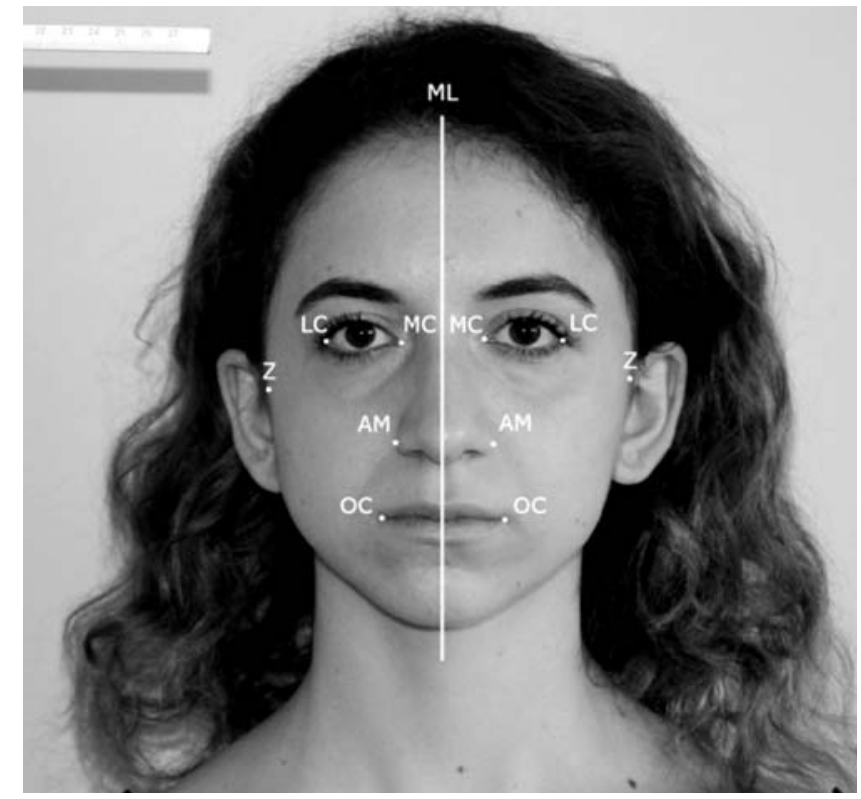

Figure 1. Anatomical landmarks for facial measurements. AM: alar margin; LC: lateral canthus; MC: medial canthus; ML: midline; OC: oral commissure; Z: zygoma. [Photograph used with permisiion from the volunteer subject]

ured and compared with the ten corresponding points on the other side of the face. This comparison was performed by dividing the measurements from the two sides, thus determining a ratio for each set of anthropometric points (LC ratio, $M C$ ratio, $A M$ ratio, $Z$ ratio, $O C$ ratio). Because the laterality of asymmetry was not important in this study, the degree of asymmetry was always determined by dividing the larger lengths by the smaller ones.

$15 \%$ of all measurements were repeated in order to evaluate the consistency of the measurements. As shown in Table 1, all measurements were found to be highly reli-

Table 1

The evaluation of consistency between the measurements.

\begin{tabular}{lccc}
\hline Measurement & Side & ICC & p \\
\hline MC & L & 0.906 & $p<0.001$ \\
LC & $R$ & 0.917 & \\
& L & 0.911 & $p<0.001$ \\
Z & $R$ & 0.965 & \\
& $L$ & 0.938 & $p<0.001$ \\
AM & $R$ & 0.963 & \\
OC & L & 0.961 & $p<0.001$ \\
& $R$ & 0.965 & \\
\hline
\end{tabular}

AM: alar margins; L: left; LC: lateral canthi; MC: medial canthi; OC: oral commissures; R: right; Z: zygoma points 
able. Consistency varied between 0.906 and 0.987 , showing a remarkable reliability of the measurements.

\section{Subjective measurements}

Subjective evaluations were conducted by seven individuals, two male and two female medical professionals and two males and one female with non-medical backgrounds. Individuals were instructed to assess each face based solely on symmetry, on neither attractiveness nor beauty. A presentation containing all photographs sequentially was prepared with commercially available Microsoft Office PowerPoint (Microsoft Corporation, 2015; Redmond, WA, USA) software. Photographs used for the presentation were unmarked and without the measurement scale. This presentation was shown to individuals onscreen. Photographs were shown for three seconds onscreen and then removed, giving the individuals two seconds to write down their assessment. A slide show with automated timing was used to minimize variation. Depending on the brief information given by researchers prior to evaluation session, individuals were asked to evaluate the faces they saw on the screen as symmetrical or asymmetrical. Individuals were asked to further evaluate the faces determined by themselves to be asymmetrical, placing the asymmetry on the upper (from middle hairline to nasion), middle (from nasion to subnasale) or lower thirds (from subnasale to menton) of the face. Individuals were given one-minute breaks after each 25 photographs and fourminute breaks after each 100 photographs. Faces evaluated by four or more than four individuals as symmetrical were considered symmetrical.

\section{Statistical analysis}

Compliance with the normal distribution of continuous variables was checked with Shapiro-Wilk test and homogeneity of groups' variances with Levene's test. When parametric test assumptions were available, two independent group means were compared using Student's t-test. If assumptions were not available, Mann-Whitney U test was used for comparisons of groups' medians. Consistency between the measurements was evaluated by the intraclass correlation coefficient (ICC). Fisher's Exact and Pearson Chi-square tests were used to analyze categorical variables. When expected frequencies were less than 5, Monte Carlo simulation method was used.

The results of the statistical analysis were expressed as number of observations (n) and percentages (\%), mean \pm standard deviation $\left(\bar{\chi} \pm S_{x}\right)$, median and minimum-maximum values [M (min-max)]. Data analyses were performed using the SPSS Statistical Package for Social Sciences (SPSS) software (version 17.0, SPSS Inc, Chicago, IL, USA). A p value less than 0.05 was considered as statistically significant.

\section{Results}

For objective evaluation, measurements from the determined anthropometric points were performed on each photograph. A rate was determined for each couple of corresponding points on each side of the face by dividing the larger length by the smaller length. In males, LC and $Z$ rates were found to be the closest to 1 . In females, LC rate was found to be the closest to 1 . In both males and females, MC rate was found to be the farthest from 1 (Table 2). No statistically significant difference concerning these rates was found between sexes $(p>0.5)$. With comparison of these rates, no subject was found to have a perfectly symmetrical face, and all subjects showed some asymmetry in one or more of their anthropometric points.

Four hundred fifty photographs were subjectively evaluated for facial asymmetry by seven individuals. $43.3 \%$ of the photographs were found to be symmetrical, whereas $56.7 \%$ were found asymmetrical. In the second stage of the subjective evaluation, individuals were asked to specify the location of the asymmetry in the faces they determined to be asymmetrical. Asymmetrical features

Table 2

Objective measurements of facial symmetry.

\begin{tabular}{|c|c|c|c|c|c|c|c|}
\hline & \multicolumn{3}{|c|}{ Male $(n=229)$} & \multicolumn{3}{|c|}{ Female $(n=221)$} & \multirow[b]{2}{*}{$p$} \\
\hline & Mean \pm SD & Median & Min-Max & Mean \pm SD & Median & Min-Max & \\
\hline AM ratio & $1.09 \pm 0.12$ & 1.06 & $1-2.43$ & $1.10 \pm 0.24$ & 1.07 & $1-4.41$ & 0.97 \\
\hline LC ratio & $1.16 \pm 0.97$ & 1.04 & $1-1.27$ & $1.06 \pm 0.14$ & 1.04 & $1-2.99$ & 0.93 \\
\hline MC ratio & $1.13 \pm 0.12$ & 1.1 & $1-2.22$ & $1.12 \pm 1.11$ & 1.10 & $1-1.72$ & 0.61 \\
\hline OC ratio & $1.09 \pm 0.23$ & 1.06 & $1-4.3$ & $1.06 \pm 0.05$ & 1.06 & $1-1.25$ & 0.18 \\
\hline$Z$ ratio & $1.06 \pm 0.12$ & 1.04 & $1-2.72$ & $1.08 \pm 0.22$ & 1.05 & $1-3.89$ & 0.45 \\
\hline
\end{tabular}

AM: alar margins; LC: lateral canthi; MC: medial canthi; OC: oral commissures; Z: zygoma points 
were found mostly in the middle third of the face (26.7\%), followed by lower third (10.1\%), upper third $(7.0 \%)$, and were found least on upper + lower thirds (1.25\%) (Table 3).

\section{Discussion}

In this study, 450 faces were objectively and subjectively evaluated by anthropometric measurements and a sevenperson jury, respectively. Our findings suggest a difference between objective measurements and subjective evaluations. Also of note, no face demonstrating symmetry for every set of anthropometric points was found during objective measurements.

To date, certain points marked on the soft tissue and the distance between these points and the midline were used for the measurement of symmetry. ${ }^{[7]}$ Midline starts from the middle of the hairline, follows the nasal bridge as a straight line, passes through the middle of the upper lip, and ends under the chin. ${ }^{[7,8]}$ Midline used in this study follows the earlier studies, traversing at least two of the trichon, nasion, subnasale and menthon points. Anthropometric points used for measurements were chosen on the basis of points used on the soft tissue and minimal difficulty while marking on the photographs. ${ }^{[3]}$

Chatrath et al. found that none of the 234 rhinoplasty patients had symmetrical faces after their measurements, and each face had asymmetrical results for at least one of the points. They also found a statistically significant difference between the regions of the face for asymmetry. Another interesting point demonstrated in their study was the subjective evaluation of the $38 \%$ of the faces as asymmetrical as opposed to a higher percentage of asymmetrical faces, which was well over $90 \%$ with objective measurements. Chatrath et al. also found a relationship between subjective perception of asymmetry and ratios derived from alar margin-midline distances. ${ }^{\left[{ }^{3}\right]}$ Shaner et al. found asymmetry of the lower face more prominent compared to asymmetries of the middle or upper face. ${ }^{[9]}$ However, Farkas found the most common and prominent asymmetry in the middle third of the face. ${ }^{[7]}$ With the subjective evaluations in our study, $58.3 \%$ of 450 faces were found to be asymmetrical. Subjective asymmetry was found most commonly in the middle third of the face (26.7\%), followed by the lower third of the face $(10.1 \%)$, and least commonly on the upper + lower thirds of the face $(1.25 \%)$. Based on these findings, it can be said that Farkas' study and ours correlate with each other. The low percentage of asymmetry on the upper third of the face (7\%) may be due to a lack of structures determining the midline. Thus, we think the best region to infer asymmetry from is the middle third of the face. Nose and alar margins may be the determining points for the observer during subjective evaluation. In previous studies, a statistically significant relationship between objective measurements of distances between alar margins and midline and the subjective perception of the face as asymmetrical were found, and it was argued that there was a relationship between objective nasal asymmetry and subjective perception of facial asymmetry. ${ }^{[3,10,11]}$

The chief reason behind the higher percentage of asymmetry on the middle third of the face is probably the nose with its vertical position on the midline. Because of this, thousands of patients complain of asymmetrical noses and request rhinoplastic surgery every year. Chatrath et al. objectively assessed patients requesting rhinoplasty and found that $97 \%$ of them had

Table 3

Subjective measurements of facial symmetry.

\begin{tabular}{|c|c|c|c|c|c|c|c|c|}
\hline \multirow[b]{2}{*}{ Evaluator } & \multicolumn{7}{|c|}{ Asymmetrical } & \multirow[b]{2}{*}{ Symmetrical } \\
\hline & Upper third & Middle third & Lower third & $\begin{array}{c}\text { Upper + } \\
\text { middle thirds }\end{array}$ & $\begin{array}{c}\text { Upper + } \\
\text { lower thirds }\end{array}$ & $\begin{array}{c}\text { Middle + } \\
\text { lower thirds }\end{array}$ & $\begin{array}{l}\text { Upper + middle + } \\
\text { lower thirds }\end{array}$ & \\
\hline 1 * & 7 & 214 & 37 & 11 & 15 & 82 & 11 & 73 \\
\hline $2^{*}$ & 14 & 107 & 33 & 1 & 4 & 1 & - & 290 \\
\hline $3^{*}$ & 35 & 96 & 42 & 11 & - & 12 & 3 & 251 \\
\hline $4^{*}$ & 15 & 88 & 9 & 3 & 3 & 5 & 3 & 324 \\
\hline 5 & 47 & 100 & 70 & - & - & - & - & 233 \\
\hline 6 & 55 & 118 & 75 & 28 & 10 & 58 & 28 & 78 \\
\hline 7 & 47 & 117 & 53 & 48 & 7 & 47 & 16 & 115 \\
\hline Total & $220(7 \%)$ & $840(26.7 \%)$ & $319(10.1 \%)$ & $102(3.2 \%)$ & $39(1.25 \%)$ & 205 (6.51\%) & $61(1.94 \%)$ & $1364(43.3 \%)$ \\
\hline
\end{tabular}

*Evaluators with medical background. 
asymmetrical faces. Their measurements were performed using anthropometric points similar to ours. In faces that were found as asymmetrical, there was no statistically significant difference between sexes concerning the distance between oral commissure points and the midline. Distances between midline and medial canthus, lateral canthus and alar margin points, however, showed less asymmetry for women compared to men. ${ }^{[3]}$ In our study, ratios derived from Z-ML distance for males, and ratios derived from LC-ML and OC-ML distances for females were found to be closest to symmetrical. Ratios derived from LC-ML distances and MC-ML distances were found to be the least symmetrical for males and females, respectively. Also of importance, no points showed statistically significant difference between sexes. In the study conducted by Chatrath et al., tragus point was also used as an anthropometric measurement point. Asymmetry was reported for some of their results $(p<0.05){ }^{[3]}$ Tragus was not used in our study, because of the difficulty of marking it correctly on photographs and the high margin of error encountered during measurements. Zygoma point, which is easier to mark on photographs, was used instead. A certain degree of asymmetry (male $1.06 \pm 0.12$, female $1.08 \pm 0.22$ ) was found for this point, along with no statistically significant difference between sexes.

This study also demonstrated individual differences for subjective evaluations. Members of the jury were asked to specify the location of the asymmetry on the faces they evaluated as asymmetrical. Individual differences were observed in this process. For example, the fourth individual evaluated only nine faces as asymmetrical on the lower third, while the sixth individual evaluated 75 faces as asymmetrical on the lower third. First individual found asymmetries on the upper thirds of seven faces, while the sixth individual found 55 faces to be asymmetrical on the upper third. The fourth individual evaluated 324 faces as symmetrical, as opposed to 73 faces evaluated as symmetrical by the first individual.
No face symmetrical for all anthropometric points was found in our objective measurements. This finding is very important for patients requesting plastic surgery procedures such as rhinoplasty out of aesthetic concerns. During the pre-operative discussions, these patients should be told that no completely symmetrical face exists naturally and a certain degree of asymmetry, however unnoticeable it is, will always persist on their faces.

\section{References}

1. Coetzee V, Greeff JM, Stephen ID, Perrett DI. Cross-cultural agreement in facial attractiveness preferences: the role of egender. PLoS One 2014;9:e99629.

2. Thornhill R, Gangestad SW. Human facial beauty: averageness, symmetry, and parasite resistance. Hum Nat 1993;4:237-69.

3. Chatrath P, De Cordova J, Nouraei SA, Ahmed J, Saleh HA. Objective assessment of facial asymmetry in rhinoplasty patients. Arch Facial Plast Surg 2007;9:184-7.

4. Sim RS, Smith JD, Chan AS. Comparison of the aesthetic facial proportions of southern Chinese and white women. Arch Facial Plast Surg 2000;2:113-20.

5. Varlik SK, Demirbas E, Orhan M. Influence of lower facial height changes on frontal facial attractiveness and perception of treatment need by lay people. Angle Orthod 2010;80:1159-64.

6. Le TT, Farkas LG, Ngim RC, Levin LS, Forrest CR. Proportionality in Asian and North American Caucasian faces using neoclassical facial canons as criteria. Aesthetic Plast Surg 2002;26:649.

7. Farkas LG, Cheung G. Facial asymmetry in healthy North American Caucasians. An anthropometrical study. Angle Orthod 1981;51:70-7.

8. Porter JP, Olson KL. Anthropometric facial analysis of the African American woman. Arch Facial Plast Surg 2001;3:191-7.

9. Shaner DJ, Peterson AE, Beattie OB, Bamforth JS. Assessment of soft tissue facial asymmetry in medically normal and syndromeaffected individuals by analysis of landmarks and measurements. Am J Med Genet 2000;93:143-54.

10. Murstein BI. Physical attractiveness and marital choice. J Pers Soc Psychol 1972; 22:8-12.

11. Zaidel DW, Cohen JA. The face, beauty, and symmetry: perceiving asymmetry in beautiful faces. Int J Neurosci 2005;115:1165-73.

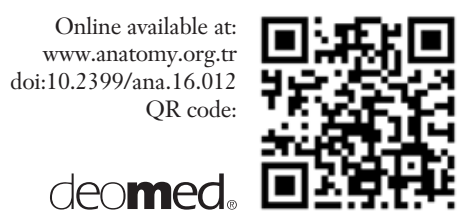

Correspondence to: Ayla Kürkçüoğlu, MD

Department of Anatomy, School of Medicine, Başkent University, 06530, Bağlıca, Ankara, Turkey

Phone: +90 3122341010

e-mail: kayla@baskent.edu.tr

Conflict of interest statement: No conflicts declared.

This is an open access article distributed under the terms of the Creative Commons Attribution-NonCommercial-NoDerivs 3.0 Unported (CC BY-NCND3.0) Licence (http://creativecommons.org/licenses/by-nc-nd/3.0/) which permits unrestricted noncommercial use, distribution, and reproduction in any medium, provided the original work is properly cited. Please cite this article as: Kürkçüoğlu A, Abbas OL, Ayan DM, Baykan R, Demirkan E, Özkan Ö, Özkubat I, Şimşek M. Comparison of objective and subjective assessments for perception of facial symmetry. Anatomy 2016;10(2):94-98. 